\title{
TFDS-3 型设备常见设备故障分析及处理方法
}

\section{Common Equipment Failures Analysis and Processing Method of TFDS-3 Type \\ 蔡博晓 \\ Boxiao Cai}

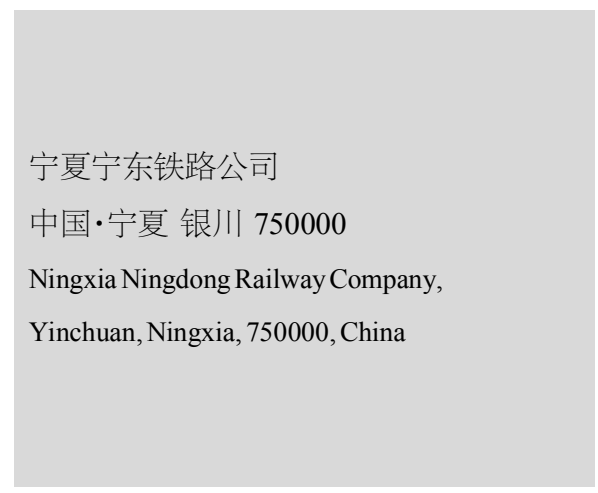

【摘要】随着科学技术的发展和普及, TFDS 货车运行故障动态图像检测系统在铁路行业 已经广泛使用, 文章对几种 TFDS-3 型设备常见的设备故障进行原因分析,并给出处理方法。 【Abstract】With the development and popularization of science and technology, TFDS truck operation fault dynamic image detection system has been widely used in the railway industry. This paper analyzes the causes of common equipment faults of several TFDS- 3 equipment and gives the processing methods.

【关键词】设备故障; 处理; 检测

【Keywords \equipment failure; handling; detection

【DOI \10.36012/etr.v2i3.1421

\section{TFDS-3 型货车运行故障动态图像检 测系统简介}

铁路货车运行故障动态图像检测系统 (简称“TFDS”) 是 一套集高速数字图像采集、大容量图像数据实时处理和精确 定位、模式识别技术于一体的智能系统, 是铁路车辆安全防范 预警系统 (5T) 的重要组成部分。系统的技术核心是轨边分布 的七台高速线阵相机，在列车通过探测站时通过精确定位技 术, 对车辆的侧架、转向架底部、车底中间部和车体进行每秒 6 万 8 千线的扫描, 合成每张 140 万像素的清晰图片传输至 列检中心服务器, 动态检车员可以在室内电脑上对这些图片 进行技术检查, 能够及时发现车辆故障并预报给前方列检确 认处理。TFDS 系统实现了列检作业从人控到机控、室外到室 内、静态检测到动态监测的变革。TFDS-3 型系统与老一代的 设备相比, 主要增加了车体侧墙部分的拍摄功能, 而且抗阳光 干扰能力更强, 在行车方向的图片无缝拼接, 增加了检车员检 车的准确性 ${ }^{[1]}$

\section{2 常见故障原因及处理方法}

\section{1 图片类}

(1)无图片信息。无图片信息的故障现象在运用中较为常 见,主要分为个别相机无图和全部相机无图两种形式。出现该 故障后, 使用 Radmin 软件远程连接至该图像采集计算机(出 现全部相机无图时,连接主图像采集计算机),检查图片采集
软件是否工作正常, 若图片采集软件报错或意外终止,则重启 图片采集软件。同时检查 $\mathrm{Z}$ 盘映射是否正常,若提示“断开网 络连接”则重新连接磁盘映射。另外, 服务器硬盘空间不足也 会导致该故障的发生，在运用中注意按时检查服务器硬盘空 间,剩余空间不足时,及时扩容。

(2)图片百叶窗。出现百叶窗故障时,需现场检查对应相机 背后触发接头是否连接正常、机柜背后对应端子排触发线是 否虚接。若连接正常, 则将故障的相机触发线并联至触发频率 相同的正常相机触发线; 并联后若过车图片恢复正常, 则判断 是车辆信息采集计算机内触发板故障, 更换触发板即可。

(3)图片过暗、白图。TFDS-3 系统使用红外线作为光源, 当 光源被阻挡、光纤老化、相机镜头污染或相机光圈太小时会发 生图片过暗的现象。图片过暗时,将相机保护盖打开,检查光 纤状态, 使用镜头清洁布清洁光源及相机镜头, 并适当将相机 光圈调大。当出现白图时说明相机箱体保护盖未打开,或保护 盖开口处有异物遮挡, 检查保护盖传动电机工作状态, 清除异 物即可解决。

\section{2 平台类}

(1)探测站接车但平台无过车信息。TFDS-3 系统采用超高 清镜头拍摄车辆图片, 单张图片占用空间大, 过车时数据传输 量激增, 对网络稳定性要求较高。当网络传输通道中的某一节 点发生波动时, 可能导致车辆信息采集计算机和服务器连接失 败,无法上传过车信息。发生故障后, 重启探测站和服务器的交 


\section{实验与研究 Experiments and Research}

换机及光纤收发器, 使用“ping”命令发送大数据包检查服务器 至车辆信息采集计算机之间的网络状态，确认是否有丢包情 况。若有丢包现象, 则排查车辆信息采集计算机和服务器之间 各传输节点间的网络通道, 确定具体故障位置, 更换相应设备。

(2)无车号信息。当列车出现无车号信息故障时,首先检查探 测站机柜内车号自动识别系统面板显示状态, 不为“0”时重启车 号主机, 等待过车测试。若故障没有排除则检查室外车号天线接 头是否紧固,依然无法排除故障的, 更换车号主机的微波单元。

(3)平台无法连接。TFDS-3 系统平台无法连接多发生在 主、备机切换后, 平台服务没有正常启动导致平台无法连接。 打开服务器的 “服务” 程序后检查各项服务运行状态, 将 “Oracle Ora Home 81 TNS Listener”和“Oracle Service TFDS” 服务启动, 将“TFDS3”服务重新启动, 平台即可恢复正常。

\section{3 其他类}

(1)网络智能插排无法连接。当探测站图像采集计算机、车 辆信息采集计算机和控制箱发生死机、无法连接时,可以使用 网络智能插排(以下简称“PDU”)进行远程掉电重启。PDU 无 法连接后, 首先使用 Radmin 软件和“ping”命令检查探测站是 否整体断网, 若探测站断网则检查网络通道问题; 若探测站其 他设备可以正常连接, 则检查 PDU 的 IP 地址是否被重置, 连
接默认的 IP 地址进入 PDU 后重新设置的 IP 地址, 若 PDU 显 示屏无法显示 IP 地址, 则 PDU 损坏, 更换 PDU。

(2)沉箱或侧箱保护门无法打开。沉箱或侧箱保护门无法 打开主要有以下几种原因: 一是传动电机故障, 先检查电机接 线有无脱落或松动, 再用电压表测量电机两个接线之间(侧箱 检查端子排保护门电机电源 CMDJ+和 CMDJ-之间的电压) 的 电压是否正常, 如有异常则更换电机。二是沉箱保护门开启路 径被阻挡。三是保护门结冰。此故障主要发生在北方, 冬天气 温低, 下雪融化结冰导致保护门冻住, 此故障可以通过设置定 时开关门功能预防, 遇大雪天气时在 TFmain 软件中开启此功 能,保护门会定期自动开关,可有效防止结冰。

\section{3 结语}

文章针对 TFDS-3 型设备在运用中产生的常见故障原因 进行分析, 结合实际情况给出处理方法。使用这些方法对设备 故障进行针对性的预防和检修, 可以提高设备检修效率, 进而 缩短设备停用时间, 保障铁路运输安全。

\section{参考文献}

[1]王德明.基于线阵扫描技术的货车故障轨边图像检测系统 [J]. 铁道车辆,2012(4):36-38.

\section{（上接第 146 页）}

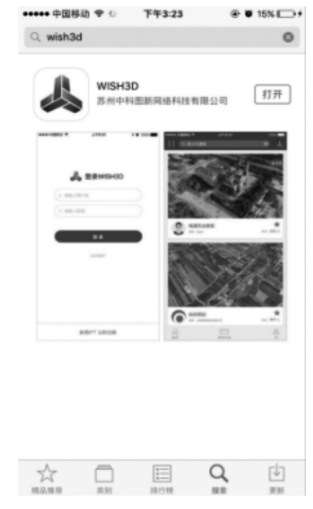

图 8 软件信息

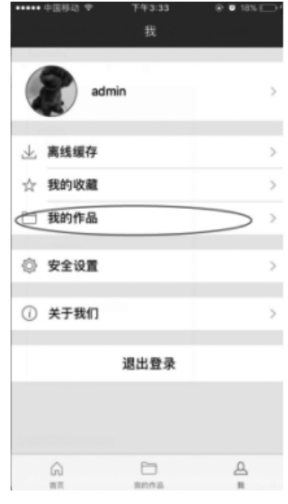

图 9 登录步骤
手机即可汶览在线的三维模型, 操作按钮分别有初始化位 置、放大、缩小、左转、右转、调整视角和全屏功能, 如图 10 所示。 很多时候, 在野外作业的情况下网络是很不稳定的, 甚至没有信 号, 这时需要在网络稳定时将模型缓存到本地, 下面简单介绍一 下。浏览页面的右上角有缓存按钮, 点击缓存, 如图 11 所示。

客户端不仅可以缓存自己上传的数据, 也可以缓存其他 用户的作品。这样一来, 上传成功的三维模型随时随地可以
用手机汶览, 实现三维模型便携化的功能。

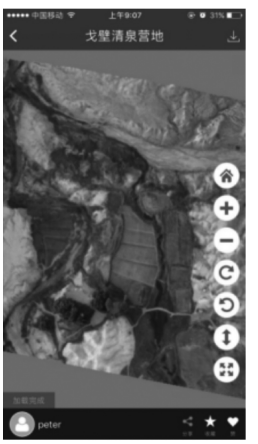

图 10 手机操作按钮

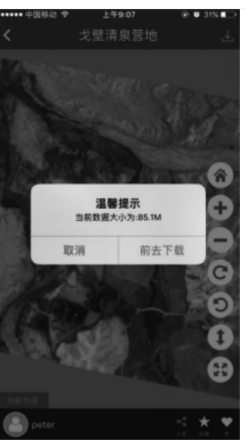

图 11 模型缓存

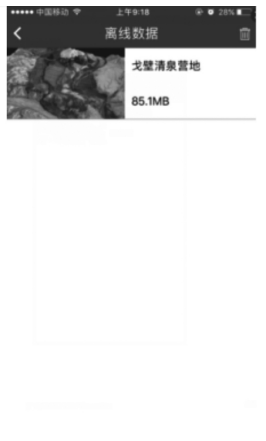

\section{4 结语}

如今, 倾斜摄影技术得以发展, 三维模型的应用也会随之 迅猛拓展, 越来越多的行业将会涉足并推动其发展。此时, 轻 便承载倾斜数据, 便捷发布三维模型的平台产品就显得尤为 重要。它适用于专业不一的用户群体, 更方便快捷地可视化 倾斜摄影三维模型, 更贴合倾斜摄影三维模型在人们生产生 活中的应用需求。 